\title{
PRODUCT STRUCTURE IN TOPOLOGICAL ALGEBRAS
}

\author{
DESMOND A. ROBBIE \\ (Dedicated to Professor A. D. Wallace on his retirement in 1973) \\ (Received 7 January, 1974) \\ Communicated by T. E. Hall
}

It is shown that every compact nonconnected semigroup (semiring) which has commuting congruences, has a nontrivial continuous homomorphic image which is iseomorphic to a direct product of finite congruence free semigroups (semirings). (This extends parts of earlier work by Kaplansky (1947) on compact rings.) It is also shown that there is a possibly finer representation but onto a product of congruence free semigroups (semirings) known only to be compact Hausdorff. A number of the techniques used evolve from work of Professor Wallace, who retired in mid-1973, and to whom this paper is dedicated.

In what follows $M$ will stand for either a semigroup or a semiring. (Following Selden (1963), (1964): A semiring is a triple, $(M,+, O)$, such that + and $\circ$ are each associative binary operations on $M$ and such that the identities-

(i) $a \circ(b+c)=(a \circ b)+(a \circ c)$

(ii) $(b+c) \circ a=(b \circ a)+(c \circ a)-$ hold

in $M$. Note that in general + is not assumed to be commutative.) When topology is involved we mean a Hausdorff topology on $M$ in which the operation(s) is (are) jointly continuous. The $\bigcirc$ will normally be omitted. A maximal congruence will always be a proper subset of $M \times M$. The words open or closed, in referring to a congruence, mean open or closed as a subset of $M \times M$ taken with the usual product topology. Whenever facts are referred to which fall into the area of algebraic semigroup theory the reader may check them in Clifford and Preston (1961). For other matters concerning topological algebra not specifically referenced the reader should find that Koch and Wallace (1954), and Wallace (1955), (1962) will suffice. We begin by stating two Universal Algebra lemmas which are of use to us.

LEMMA 1. If $\sigma$ and $\rho$ are congruences on $M$, such that $\rho \supseteq \sigma$, then denoting the canonical map from $M$ to $M / \sigma$ by $\theta$, it follows that

$$
\rho=(\theta \times \theta)^{-1}[(\theta \times \theta)(\rho)] \text {. }
$$

(C) Copyright Australian Mathematical Society 1975

Copyright. Apart from any fair dealing for scholarly purposes as permitted under the Copyright Act, no part of this JOURNAL may be reproduced by any process without written permission from the Treasurer of the Australian Mathematical Society. 
Proof. Omitted.

LeMMA 2. If $\sigma$ and $\rho$ are congruences on $M$ such that $\rho \supseteq \sigma$, and if $\rho$ is maximal, then, denoting the canonical map from $M$ to $M / \sigma$ by $\theta$, we have that $(\theta \times \theta)(\rho)$ is a maximal congruence on $M / \sigma$.

Proof. Omitted. Follows from Lemma 1.

Before proceeding further we remark that although some other parts of the paper relate also to general algebras, and not just to semigroups or semirings, the main results are not likely to hold in such general settings due to difficulties in establishing the presence of an open and maximal congruence in more general cases.

From now on-we will be interested in the following property which not all semigroups or semirings have but which is possessed by all of them which have commuting congruences, which includes groups and rings. (See final page).

Property $X$. Whenever $J$ is a maximal congruence on $M$, and $B$ is a proper congruence on $M$ such that $B \nsubseteq J$, then every $J$ class meets every $B$ class.

Lemma 3. Any $M$ in which congruences commute has Property $X$.

Proof. As is well known, when congruences commute the join of any pair of congruences is their composition. Thus if $J$ is maximal and $B \nsubseteq J, B$ proper, then we have that

$$
M \times M=J \circ B=B \circ J .
$$

Thus if $x$ is in a $J$-class $[x]_{J}$ and $y$ is in a $B$ class $[y]_{B}$ then there exists $c$ such that $(x, c) \in J$ and $(c, y) \in B$.

This means that

$$
[x]_{J} \cap[y]_{B} \neq \square .
$$

In fact, we only need Property $X$ and not that congruences commute. However, as there has been considerable investigation of algebras in which congruences commute (see Cohn (1965) for references) we felt it better to state our requirement in that way. We are pleased to acknowledge comments on this from P. Jones of Monash University, Mathematics Department.

COROllary to Lemma 3. Groups and rings have Property $X$.

Proof. As may be checked groups and rings have commuting congruences.

Lemma 4. If $M$ has Property $X$, so does any homomorphic image of $M$.

Proof. Suppose we have $\theta: M \rightarrow M^{\prime}$, and $M$ has Property $X$. As is well known $M^{\prime}$ is isomorphic to $M / \sigma$ where $\sigma$ is the canonical congruence induced 
by $\theta$. Thus we consider whether $M / \sigma$ has Property $X$. By abuse of notation we let $\theta$ also denote the canonical factor map from $M$ to $M / \sigma$.

Suppose now that $J^{\prime}$ is a maximal congruence on $M / \sigma$ and that $B^{\prime}$ is a proper congruence on $M / \sigma$ with $B^{\prime} \ddagger J^{\prime}$.

Set $J=(\theta \times \theta)^{-1}\left(J^{\prime}\right)$ and $B=(\theta \times \theta)^{-1}\left(B^{\prime}\right)$, congruences on $M$, each one of which includes $\sigma$.

Now if there exists $\tilde{J}$ a proper congruence on $M$ such that $\tilde{J} \supsetneqq J$ we must have that $(\theta \times \theta)(\tilde{J})$ is a proper congruence on $M / \sigma$ and which properly contains $J^{\prime}$, by a double application of Lemma 1 . However this contradicts the maximality of $J^{\prime}$. Hence $J$ is maximal in $M$. Also $B$ is a proper congruence on $M$ by a similar argument. Further it is clear that $B \nsubseteq J$. Now for any $J^{\prime}$ class $[x]_{J}$, in $M / \sigma$ and any $B^{\prime}$ class $[y]_{B^{\prime}}$ in $M / \sigma$ their inverse images under $\theta$ are a $J$ class and a $B$ class respectively and so, by Property $X$ in $M$, must meet. Then clearly the image under $\theta$ of this intersection must be nonempty and included in $[x]_{J^{\prime}} \cap[y]_{B^{\prime}}$, so that we have Property $X$ in $M / \sigma$. Then by the isomorphism between $M^{\prime}$ and $M / \sigma, M^{\prime}$ has Property $X$.

Definition. $M$ is said to be congruence free if the only congruences on it are $M \times M$ and $\Delta$.

Lemma 5. Let $M$ be compact, and have Property $X$. Let $J_{1}, \cdots, J_{n}$ be closed, maximal congruences on $M$, such that, if $n>1, J_{1} \cap \cdots \cap J_{t} \ddagger J_{t+1}$, for each $t=1, \cdots, n-1$. Then with the canonical topologies, and the canonical map, we have

$$
M /\left(J_{1} \cap \cdots \cap J_{n}\right) \stackrel{t}{\cong} M / J_{1} \times \cdots \times M / J_{n} .
$$

Each of the $M / J_{i}$ is a congruence free compact algebra of the same type as $M$, and, if $J_{i}$ is open, then $M / J_{i}$ is finite and discrete.

Proof. By Sierpinski's Lemma, the following diagram may be completed as shown,

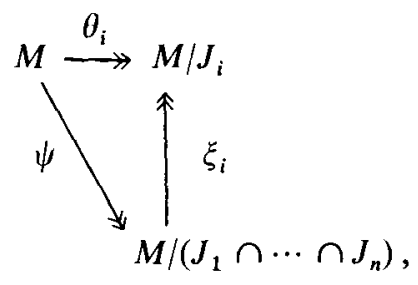

by the canonical map $\xi_{i}$ which is a continuous homomorphism. As is well known, each of the spaces, with the quotient topology, is compact Hausdorff, and the multiplication (or addition) induced in the canonical way is bicontinuous. When $J_{i}$ is open, then, since $M$ is compact, there is only a finite number of congruence classes, so that $M / J_{i}$ is finite, and hence has the discrete topology. Clearly, $M / J_{i}$ 
is always congruence free, for, if not, then a proper nontrivial congruence on $M / J_{i}$ would imply one on $M$ and which contained $J_{i}$, thus contradicting the maximality of $J_{i}$.

Now consider:

$$
\pi \xi_{i} \quad M /\left(J_{1} \cap \cdots \cap J_{n}\right) \stackrel{\pi \xi_{i}}{\stackrel{i=1, \cdots, n}{\longrightarrow}} M / J_{1} \times \cdots \times M / J_{n},
$$

where $(i=1, \cdots, n)(x)=\left(\xi_{1}(x), \cdots, \xi_{n}(x)\right)$.

Since our product has the canonical product topology induced by the $M / J_{i}$, and since each $\xi_{i}$ is continuous, it is well known that $\Pi_{i=1, \ldots, n} \xi_{i}$ is continuous and a homomorphism. That it is 1-1 is obvious. The only thing to show is that it is onto. It $n=1$, it is trivial. If $n=2$, then as $J_{1} \notin J_{2}$, by hypothesis, then by Property $X$, each $J_{2}$ class meets each $J_{1}$ class which means that our map is onto. Now, since for $3 \leqq m \leqq n J_{1}, \cdots, J_{m-1}$ has the property $J_{1} \cap \cdots \cap J_{t} \ddagger J_{t+1}$ for each $t=1, \cdots, m-2$, then by the induction assumption

is onto.

$$
M /\left(J_{1} \times \cdots \times J_{m-1}\right) \stackrel{\substack{i=1, \ldots, m-1 \\ i}}{\longrightarrow} M / J_{1} \times \cdots \times M / J_{m-1}
$$

Now $M /\left(J_{1} \times \cdots \cap J_{m}\right) \simeq M /\left(\left(J_{1} \cap \cdots \cap J_{m-1}\right) \cap J_{m}\right)$ and since $J_{1} \cap \cdots$ $\cap J_{m-1} \ddagger J_{m}$, by hypothesis, then by Property $X$ again

$$
\begin{aligned}
M /\left(\left(J_{1} \cap \cdots \cap J_{m-1}\right) \cap J_{m}\right) & \stackrel{\text { onto }}{\longrightarrow} M /\left(J_{1} \cap \cdots \cap J_{m-1}\right) \times M / J_{m} \\
& \stackrel{\text { onto }}{\longrightarrow} M / J_{1} \times \cdots \times M / J_{m-1} \times M / J_{m} .
\end{aligned}
$$

So altogether we have that our map is onto for $3 \leqq m \leqq n$ if it is for $m-1$.

Since our map is onto for $m=2$ we have by finite induction that it is onto for $m=n$.

This completes the proof of the Lemma.

Further Definitions. Let $M$ be compact, and let $T(M)$ be the set of open and maximal congruences on $M$, if there are any; otherwise, $T(M)$ is the singleton set containing $M \times M$ as its only element. We define the radical of $M$, by

$$
R(M)=\bigcap T(M) .
$$

If $R(M)=\Delta$, we say that $M$ is semisimple. We note that $R(M)$ is closed, and so $M / R(M)$ is again a compact topological algebra. We further note that, if we replace the word "open" above by "closed", $T(M)$ by $\tilde{T}(M)$ and $R(M)$ by $\tilde{R}(M)$, the weak radical, our definitions and remarks still make sense. If $\tilde{R}(M)=\Delta$ we say that $M$ is weakly semisimple.

LEMma 6. If $M$ is compact, then $R(M / R(M))=\Delta$. (The same is true for $\widetilde{R}(M)$ and the proof is simpler). 
Proof. If $T(M)=\{M \times M\}$ then the proof is trivial. Otherwise there is at least one, open and maximal, congruence on $M$. Then let $\rho \in T(M)$. Denoting as usual the canonical map from $M$ to $M / R(M)$ by $\theta$, the set $(\theta \times \theta)(\rho)$ is an open and maximal congruence on $M / R(M)$. This is because: (i) By Lemma 2 above, $(\theta \times \theta)(\rho)$ is a maximal congruence on $M / R(M)$; and: (ii) Each $(\theta \times \theta)(\rho)$ class, say $A$, has for its preimage a $\rho$ class $A^{\prime}$, which must then be open and so $A$ is open. Now choose an arbitrary pair $(p, q) \in[M / R(M)] \times[M / R(M)]$ so $p \neq q$. We have $p=\theta(a), q=\theta(b)$ for some $a$ and $b$ in $M$, and also $(a, b) \notin R(M)$. Thus, there exists an open and maximal congruence, say $J$, on $M$ such that $(a, b) \notin J$. Then $(\theta(a), \theta(b)) \notin(\theta \times \theta)(J)$ since, by Lemma $1, J=(\theta \times \theta)^{-1}[(\theta+\theta)$ $(J)]$. However, as already noted $(\theta \times \theta)(J)$ is an open and maximal congruence on $M / R(M))$ and thus $R(M / R(M)=\Delta$.

LEMMA 7. Any congruence $\rho$, on a compact $M$, which is maximal in the set $F$ (assumed nonempty) of open proper congruences on $M$, is a maximal congruence on $M$. That is, $\rho$ is an open and maximal congruence on $M$.

Proof. Since $\rho$ is open it is also closed. Since $M$ is compact, $M / \rho$ is finite and has the discrete topology. So then $M / \rho \times M / \rho$ is also finite, and also has the discrete topology. Suppose that $M \times M \supsetneqq \tau \supsetneqq \rho$, and $\tau$ is a congruence on $M$. Then, denoting the canonical map from $M$ to $M / \rho$ by $\theta$, we have that $(\theta \times \theta)(\tau)$ is an open congruence on $M / \rho$ because there all congruences are open, since we have the discrete topology. Then, by Lemma 1 , we have that $\tau=(\theta \times \theta)^{-1}[(\theta \times \theta)$ $(\tau)]$, and $\tau$ is open because $\theta \times \theta$ is continuous, since $\theta$ is continuous. This contradicts the maximality of $\rho$ in $F$, and thus $\rho$ is not only maximal in the set $F$, but is a maximal congruence on $M$.

LEMMA 8. If $M$ is compact, and has an open and proper congruence $\sigma$ on it, then it has a congruence $\rho$ on it which is both a maximal congruence, and an open congruence. (We note that $\rho$ is of course also closed.)

Proof. By a simple application of Zorn's Lemma, there is at least one congruence on $M$, say $\rho$, which is maximal in the set of open and proper congruences on $M$, and contains $\sigma$. Then, by Lemma $7, \rho$ is also a maximal congruence on $M$.

LEMMA 9. For $(R,+, O)$ a semiring, $T$ a subsemiring of $R$ (i.e. a subset of $R$ algebraically closed under + and $\bigcirc)$, if $R \supseteq A \neq \square$, then the set $D_{T}(A)$ described below is the smallest double $T$-ideal (i.e. subset of $R$ which contains all sums and products resulting from elements of itself and of $T$ ) of $R$ which contains $A$.

$$
D_{T}(A)=\bigcup\left\{T_{i}+T_{j} A T_{k}+T_{l}: i, l \in\{1,2\}, j, k \in\{2,3\}\right\}
$$


where $T_{1}$ is a formal additive identity on $R, T_{2}=T$, and $T_{3}$ is a formal multiplicative identity on $R$.

ProOF. Omitted.

Note that this lemma appears first in Robbie (1970), and later. in the special case $T=M$ in Bertman and Selden (1973). We will need it in a different special case namely, $R=M \times M$ and $T=\Delta$, when $M$ is itself a semiring.

In fact the ability to write out $D_{T}(A)$ explicitly with finitely many unions of finitely many additions and multiplications is the crucial part in obtaining our main result and is the reason why we stated earlier that it is doubtful if the main result would be extendable to general algebras.

LEMMA 10. Let $(R,+, O)$ be a compact semiring, $T$ a closed subsemiring of $R, K_{T}(R)$ a double $T$ ideal of $R$ (when $T=M$ first shown to exist by Selden (1963), and $U$ an open subset of $R$ which contains $K_{T}(R)$. Then the union, $J$, of all double T-ideals contained in $U$, is an open subset of $R$.

Proof. Omitted. Exactly as in earlier work of Koch and Wallace (1954) given Lemma 9 above.

We remark in passing that from Lemma 10 come maximal double $T$-ideals, which are open, as first noted in Robbie (1970) and later, case $T=M$, in Bertman and Selden (1973).

In this paper we use Lemma 10 in the special case $T=\Delta, R=M \times M$, and case $K_{\Delta}(M \times M)=\Delta$, so that the lemma applies for any open subset of $M \times M$ which contains $\Delta$.

LEMMA 11. Let $(M,+, \circ)$ be a compact totally disconnected semiring. Then there exists, $C$, an open and closed (clopen) proper subset of $M \times M$, which contains $\Delta$ and is a congruence on $M$. (In fact every open subset of $M \times M$ which contains $\Delta$, contains such a congruence.)

Proof. The proof is merely mutatis mutandis from that of Numakura (1957) who gives the semigroup case. The difference being the need to invoke Lemma 10 in the manner mentioned above plus some other minor changes.

We also note in passing that this lemma can be used to show that each compact totally disconnected semiring is a strict projective limit of finite discrete semiring.

Corollary to Lemma 11. Any nonsingleton, compact, totally disconnected $M$ has a congruence on it which is both an open congruence and a maximal one.

ProOF. By Lemma 11 and the similar result for semigroups by Numakura (1957), plus the application of Lemma 8, the result follows immediately. 
LEMMA 12. Any nonsingleton, compact, totally disconnected $M$ has a nontrivial semisimple homomorphic image, and a nontrivial weakly semisimple image.

Proof. Immediate from Lemma 6 and the Corollary to Lemma 11.

LEMMA 13. If $I$ is a set of congruences on $M$ such that $\cap I=\Delta$, then $I$ may be well ordered such that, for some $I^{\prime} \subseteq I$ with the well ordering induced from that on $I$, we will have the following two properties satisfied:

(i) $\cap I^{\prime}=\Delta$.

(ii) If $T_{\alpha_{1}}^{\prime}, \cdots, T_{\alpha_{n}}^{\prime}$ is any finite set, with two or more members from $I^{\prime}$, arranged in increasing order by the well order on $I^{\prime}$, then

$$
\left(T_{\alpha_{1}}^{\prime} \cap \cdots \cap T_{\alpha_{n-1}}^{\prime}\right) \subseteq T_{\alpha_{n}}^{\prime} .
$$

Proof. Well order $I$, and index with an ordinal $k$. Choose a subset of $I$ as follows:

$$
\text { if } k=0, I^{\prime}=I=\left\{T_{0}\right\} \text {. }
$$

Otherwise, define a function $f: I \rightarrow\{0,1\}$ by the following procedure. Set $f\left(T_{0}\right)=1$, and let $\alpha$ be any ordinal such that $k \geqq \alpha>0$. Then let

$$
B_{\alpha}=\left\{\beta: \beta<\alpha \text { and } f\left(T_{\beta}\right)=1\right\}\left(\neq \square \text { as } 0 \in B_{\alpha}\right) \text {. }
$$

If, for every finite subset of $B_{\alpha}: \beta_{1}, \cdots, \beta_{n}$ ( $n$ a natural number) in increasing order, we have $T_{\beta_{1}} \cap \cdots \cap T_{\beta_{n}} \notin T_{\alpha}$, then we set $f\left(T_{\alpha}\right)=1$, otherwise, we set $f\left(T_{\alpha}\right)=0$.

We note that we do have a function. For, if not, choose the least member $T_{\alpha}$ of $I$ for which our function is not defined. This is possible since $I$ is well ordered. Certainly $\alpha>0$, so that $B_{\alpha}$ exists and is not empty. Then we choose $f\left(T_{\alpha}\right)$ unambiguously as above, which is a contradiction. So $f$ is defined on all of $I$. A similar argument shows that our $f$ is uniquely defined. Now define $I^{\prime}$, by $I^{\prime}=f^{-1}(1)$. We must show that $I^{\prime}$ has properties (i) and (ii). We note first that the order induced on $I$ is a well order on $I^{\prime}$. (i) If $x \neq y$, and $(x, y) \in T_{\alpha^{\prime}}$ for each $T_{\alpha^{\prime}} \in I^{\prime}$, then there exists $T_{r^{\prime \prime}} \in I \backslash I^{\prime}$ such that $(x, y) \notin T_{\alpha^{\prime \prime}}$ (since $\cap I=\Delta$ ). Now, if $T_{\alpha^{\prime \prime \prime}}$ is the least member of $I$ with this property, consider $\boldsymbol{B}_{\alpha^{\prime \prime \prime}}=\left\{\beta ; \beta<\alpha^{\prime \prime \prime}\right.$ and $\left.f\left(T_{\beta}\right)=1\right\}$. Now $(x, y) \in \cap_{\beta \in B_{\alpha^{\prime \prime \prime}}} T_{\beta} \neq T_{\alpha^{\prime \prime \prime}}$, and so certainly, for any finite set $T_{\beta_{1}}, \cdots, T_{\beta_{n}}, \beta_{i} \in B_{\alpha^{\prime \prime \prime}}$, we have $T_{\beta_{1}} \cap \cdots \cap T_{\beta_{n}} \neq T_{\alpha^{\prime \prime \prime}}$. But then, $f\left(T_{\alpha^{\prime \prime \prime}}\right)=1$, which is a contradiction, and so $\cap I^{\prime}=\Delta$.

Now (ii). Suppose we choose a finite set $T_{\alpha_{1}^{\prime}}, \cdots, T_{\alpha_{n}}^{\prime}$ from $I^{\prime}$, and that $T_{\alpha_{1}} \cap \cdots \cap T_{\alpha_{n-1}^{\prime}} \subseteq T_{\alpha_{n}^{\prime}}$. Now $\left\{\alpha_{1}^{\prime}, \cdots, \alpha_{n-1}^{\prime}\right\} \subseteq B_{\alpha_{n}^{\prime}}^{\prime}$ and so $f\left(T_{\alpha_{n}}^{\prime}\right)=0$, and this contradicts $f\left(T_{\alpha_{n}}^{\prime}\right)=1$. So we have established both required properties.

THEOREM 1. If $M$ has Property $X$ and is compact semisimple, then $M$ is iseomorphic to a direct product of finite congruence free algebras of the same type as $M$, with the discrete topology on each one (the canonical map, and 
canonical product topology being used). (If $M$ has property $X$, and is compact weakly semisimple, then we must substitute compact Hausdorff, for finite with the discrete topology.)

Proof. If $I$ is the set of open and maximal congruences on $M$, we are given that $\cap I=\Delta$. Then, by Lemma 13 above, we may choose from $I$ a well ordered set $I^{\prime}$ with the property that, for any finite subset $T_{\alpha_{1}}, \cdots, T_{\alpha_{n}}$ from $I^{\prime}$, we have $T_{\alpha_{1}} \cap \cdots \cap T_{\alpha_{n-1}} \neq T_{\alpha_{n}}$.

Fixing $n=\bar{n}$, we may apply this fact again to each of the sets $\left\{T_{\alpha_{1}}, \cdots, T_{\alpha_{t}}\right\}$, $t=2, \cdots, \vec{n}-1$. So that, denoting $T_{\alpha_{i}}$ by $J_{i}, i=1, \cdots, \bar{n}$, we have that $\left\{J_{1}, \cdots, J_{\bar{n}}\right\}$ is a set of maximal and open congruences on $M$ such that $J_{1} \cap \cdots \cap J_{s} \ddagger_{s+1}$, $s=1, \cdots, n-1$. Then by Lemma 5 ,

$$
M /\left(J_{1} \cap \cdots \cap J_{\bar{n}}\right) \stackrel{!}{\cong} M / J_{1} \times \cdots \times M / J_{n},
$$

or,

$$
M /\left(T_{\alpha_{1}} \cap \cdots \cap T_{\alpha_{\bar{n}}}\right) \stackrel{t}{\cong} M / T_{\alpha_{1}} \times \cdots \times M / T_{\alpha_{\bar{n}}} .
$$

Thus we have a homomorphism.

$M \rightarrow M / T_{a_{1}} \times \cdots \times M / T_{a_{\bar{n}}}$ for each $\bar{n}$ a natural number, and each $T_{\alpha_{1}}, \cdots, T_{a_{\bar{n}}}$ in $I^{\prime}$. So the canonical map $M \rightarrow{ }_{T_{\gamma}} \Pi_{\in I}, M / T_{\alpha}$ attains at least one point with any finite number of predetermined coordinates. By the nature of the product topology on the right, the image of $M$ is dense in the product. (This is true even if the $T_{\alpha}$ are merely closed.) Moreover, as is well known, the canonical function, namely ${ }_{T_{\alpha}} \Pi_{\in I}, 0_{\alpha}$ (where $0_{\alpha}: M \rightarrow M / T_{\alpha}$ ) is continuous, because each of the separate $\theta_{\alpha}$ is continuous. So, since $M$ is compact, $\left({ }_{T_{\alpha}} \Pi_{\in I}, \theta_{\alpha}\right)(M)$ is closed and, being dense in the product, must be equal to it.

We acknowledge with pleasure assistance received from D. Hajek, now of Mayaguez, during discussions on this part.

THEOREM 2. Every compact nonconnected semigroup (semiring) which has commuting congruences, has a nontrivial continuous homomorphic image which is iseomorphic to a direct product of finite congruence free semigroups (semirings). It is also the case that there is a possibly finer representation but onto a product of congruence free semigroups (semirings) known only to be compact Hausdorff:

Proof. Firstly, one obtains a continuous homomorphic image which is nonsingleton and totally disconnected by factoring modulo the congruence with classes which are the connected components. Now as $M$ has commuting congruences it has Property $X$, by Lemma 3. Thus our totally disconnected image also has Property $X$, by Lemma 4 . Then factoring our present image modulo its radical (or the weak radical) we obtain a further contınuous homomorphic image which still has Property $X$, by Lemma 4, and which, by Lemma 12, will be semisimple 
(weakly semisimple) and nontrivial. Since our image is compact as well Theorem 1 applies and we are finished.

We note that there must be an abundance of semigroups and semirings which are compact totally disconnected and which have Property $X$. This is because, apart from the special cases of groups and rings, any direct product of finite congruence free objects has Property $X$ and then, taking any continuous homomorphic image and factoring over its connected component congruence, we would again obtain a compact totally disconnected object with Property $X$. We can see no immediate reason why an object obtained in this way should not be fairly complicated. Of course if it is nontrivial then it ill in its turn have a nontrivial image which is once again a direct product of finite congruence free objects.

How far this work may be extended to the locally compact case and under what further conditions remains very much an open question.

\section{References}

M. Bertman and J. Selden (1973), 'Double ideal in compact semirings', J. Austral. Math. Soc. 15, 123-128.

A. H. Clifford and G. B. Preston (1961), 'Algebraic theory of semigroups' (Surveys, 7 Amer. Math. Soc. 1961).

P. M. Cohn (1965), Universal Algebra (Harper and Row, New York, Evanston and London, and John Weatherhill, Inc., Tokyo, 1965).

I. Kaplansky (1947), 'Topological rings', Amer. J. Math. 69, Page 164, Theorem 16.

R. J. Koch and A. D. Wallace (1954), 'Maximal ideals in compact semigroups', Duke Math.J. 21, 681-686.

K. Numakura (1957), 'Theorems in compact totally disconnected semigroups and lattices', Proc. Amer. Math. Soc. 8, 623-626.

D. A. Robbie (1970), Some theorems on binary topological algebras (O-P Book, University Microfilms, Inc., Ann Arbor, Michigan, 1970).

J. Selden (1963), Theorems on topological semigroups and semirings, (O-P Book, University Microfilms, Inc., Ann Arbor, Michigan, 1963).

J. Selden (1964), 'A note on compact semirings', Proc. Amer. Math. Soc. 15, 882-886.

A. D. Wallace (1955), 'On the structure of topological semigroups,' Bull. Amer. Math. Soc. 61, 95-112.

A. D. Wallace (1962), 'Relative ideals in semigroups, I (Faucett's Theorem)', Coll. Math. 9, $55-61$.

Department of Mathematics

Adelaide College of Advanced Education

Adelaide, South Australia. 